\title{
An ITS architecture specification - The FOTsis project experience
}

\section{Especificación de arquitecturas ITS - La experiencia del proyecto FOTsis}

Alfonso, J. - Sánchez, N. - Menéndez, J.M. - Cacheiro, E.

Abstract: The recent continuous development of Cooperative ITS has resulted in several initiatives which focus on different parts of the Cooperative environment landscape. The FOTsis project focuses on the infrastructure side of the Cooperative environment and will deploy and test 7 services designed to maximise the benefits of the integration of the road operator and infrastructure-based information providers into the ITS environment. This paper describes the current status of the project and focuses on the road safety approach within the project: safety services and safety impact assess- 
ment. The outlook on the project's next steps is given in the last section of the paper.

Keywords: Field Operational Test, Cooperative ITS, ITS Services, Architecture.

Resumen: El constante desarrollo reciente de los ITS Cooperativos ha resultado en diferentes iniciativas que se centran en distintos aspectos del entorno Cooperativo. El proyecto Europeo FOTsis perteneciente al FP7 gira alrededor de la infraestructura de este entorno. El proyecto tiene como objetivos básicos desplegar y validar 7 servicios que han sido diseñados para maximizar los beneficios de la integración de diferentes entidades basadas en la infraestructura en el paisaje ITS: el operador de infraestructura, proveedores de datos externos, entre otros. Este artículo describe el estado actual del proyecto, destacando los aspectos de seguridad vial dentro del mismo: los servicios FOTsis de seguridad y el análisis del impacto en términos de seguridad. Al final del artículo se da una visión general de los próximos pasos en el proyecto.

Palabras clave: Campo operacional de pruebas, ITS Cooperativos, servicios ITS, arquitectura. 


\section{Introduction}

New communication technologies open seemingly endless possibilities to the generation of new services in basically every field of the society. With the emergence in recent years of first, the concept and subsequently the practical deployment of V2X communications, the problem with road transportation is maybe not so much one of possibilities as it is one of selecting the services' design and technologies most appropriate for the given objectives. Additional consideration is given then to the conditions which ensure a stable and consistent development and deployment framework which ensures in turn homogeneity in the implementation and provision of services, both for the users and the service providers.

It is in this sense perhaps that the driving force dictating the requirements for the architectures supporting the advanced road transport services has shifted from purely the technological aspects to areas closer to 'Quality of Service' and 'Quality of Experience'. Nowadays, there is a greater knowledge about the user's requirements and how to specify systems that can enhance the user's experience of a service through those requirements. ITS Services are one of the approaches that can promote of advancement of technological solutions with the goal of attaining user's requirements.

In order to assess the impact of the services deployed, FOT (Field Operational Test) projects not only must include stages for specification and deployment of the services themselves, but additionally address the even more important issue of testing and evaluating the services in terms of overall objectives such as improving traffic effi- 
ciency, road safety and transport sustainability. In the broadest sense, impact analyses addressing these objectives cannot be limited to traffic data or incident data. Road safety is a particularly sensitive issue, and the analysis of any C-ITS service in terms of safety is a complex task. Several assessment methodologies are considered that include the necessary requirements in relation to subjective and objective data collection, analysis tools and links with related parameters such as data accuracy, user's service uptake and broader socio-economic analysis of the deployment of the service.

\subsection{The FOTsis project}

There are several approaches to the design of ITS services, depending on who are the final users, what are the available sources of data, or what are the means to notify the final processed information to the users. The current focus of the Cooperative ITS services is on the mobile entities of the road environment, therefore pushing especially concepts such as the V2V communications stack, development of on-board vehicle sensors and devices, and in general a very vehicle-oriented service design.

However, there are, amongst others, two factors that have to be taken into account when looking at future trends in ITS Services. One is the increasing importance of the idea of transport design towards specific goals of efficiency in mobility, safety and sustainability, which require a different approach to data collection than exclusively road transport environments, and the other one is altogether a more practical one; there is a large amount of resources of different kind already deployed by the road operators which might not be fully utilised in current $\mathrm{V} 2 \mathrm{~V}$-oriented services.

The FOTsis project takes up this second factor and develops the general concept of pushing the involvement of the road infras- 
tructure in all the stages of ITS service provision. FOTsis will specifically test the road infrastructure's capability to incorporate the latest cooperative system technology at nine test sites in four countries, as can be seen in Figure 1, providing a set of seven comprehensive service entities focusing on traffic safety and efficiency improvements.

- Service $1 \quad$ Emergency management

- Service 2 Safety incident management

- Service 3 Intelligent congestion control

- Service 4 Dynamic route planning

- Service 5 Special vehicle tracking

- Service 6 Advanced enforcement

- Service 7 Infrastructure safety assessment

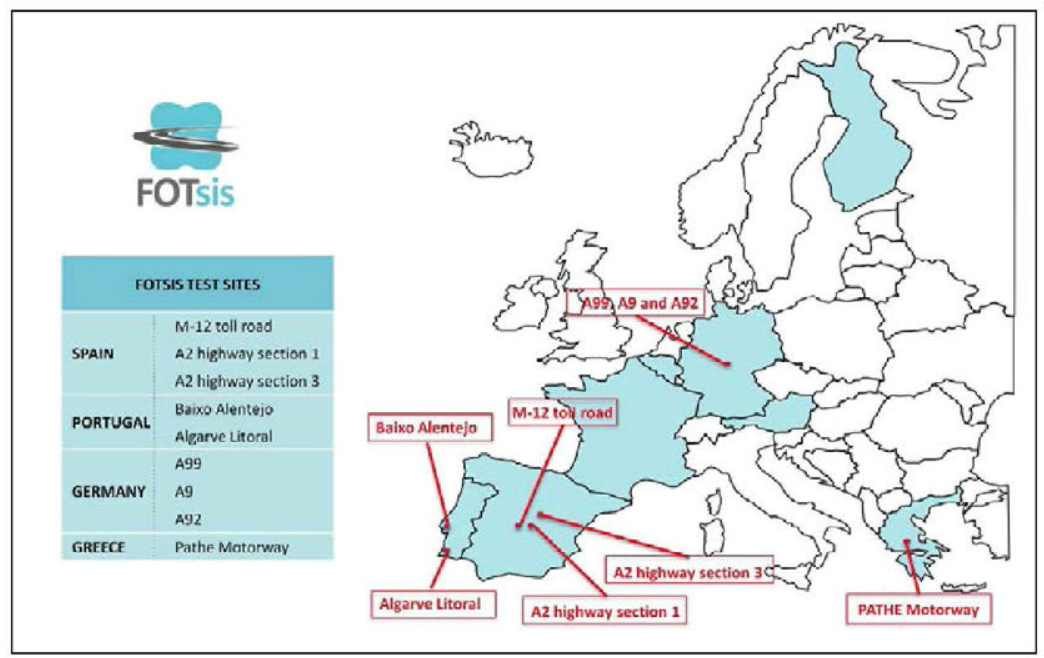

Figure 1: FOTsis participating countries and test sites. 
In the first stage of the definition of the architecture, the varied nature of the services and their different requirements and needs in terms of data collection, processing and notification to the relevant users is taken into account to provide a set of requirements for the architecture to be instantiated and implemented.

After the services have been deployed, an evaluation of their performance will take place, addressing their improvements in terms of the above mentioned objectives of transport efficiency, road safety and sustainability. Several initiatives have been analysed in order to carry out in FOTsis the most appropriate evaluation method to obtain the most significant results. While FOTsis services $\mathrm{S} 1$ and $\mathrm{S} 2$ can be considered to be essentially safety-oriented, all 7 services will be subject to the safety impact assessment.

\section{The FOTsis safety services}

\subsection{Service 1 - Emergency management}

The FOTsis Emergency Management Service is based on the detection of an emergency situation and extending the current concept of the standardised e-Call to integrate emergency calls which could come from an emergency application in the user's mobile phone or from the road operator using its own incident detection systems.

After the initial validation of the incident, further information (e.g. a map with guidance to the incident or pictures about the incident) can be collected using different means and will be shared in real-time amongst the actors involved in the resolution of the incident (road operator, emergency authorities, road operator response teams and emergency response teams) by means of deployed applications at the respective management centres or on-board tablet PCs. 


\subsection{Service $\mathbf{2}$ - Safety incident management}

The FOTsis Safety Incident Management is based on the idea of extending the advanced incident data collection and emergency resources management established in Service 1 to the notification of this information to the road users. Information about the incidents in real time is shown via an application in the user's smartphone or tablet.

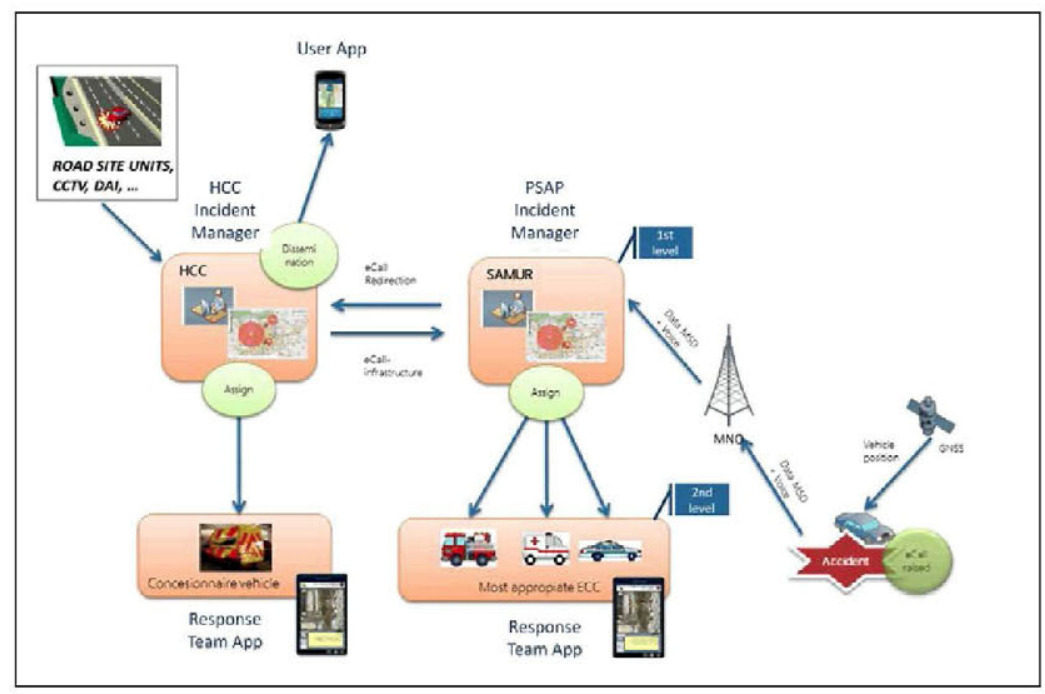

Figure 2: FOTsis integrated Service1/Service2 diagram.

Working in conjunction, FOTsis Service $1 \&$ Service 2 aim to improve response times and the quality of the response to any given incident on the road. Improved awareness on the part of all the entities in the road environment will yield an expected result in traffic mobility and better allocation of emergency resources. 


\section{The FOTsis architecture}

\subsection{The overall FOTsis environment}

It is important to highlight the fact that the FOTsis perspective on ITS services integrates a number of different entities, each with its own role to play in the provision of the service. In the description of the two FOTsis services above, besides the road user and road operator, these entities may include traffic management centres, emergency services and management authorities, weather and map data providers, etc. In fact, one of the objectives of the project to establish the framework so that the services can take full advantage of the inclusion of these entities in the services.

\subsection{The FOTsis communications architecture}

The basis of the FOTsis architecture in terms of communications is the ITS Station Reference Architecture (ISO 21217:2010). The ITS-Station architecture provides a communications framework and deployment methodology to ensure interoperability between the different Cooperative systems and services initiatives.

Its elements have been instantiated whenever necessary, according to the requirements of the selected FOTsis services, starting with the basic deployment connectivity structure, which implies both a long distance link with the user from the Central ITSStation at the infrastructure side of the service and a short distance link by making use of the roadside communication facilities provided by the road operator. Together with the basic links with external data providers and external service and infrastructure entities, the basic connectivity scenarios resulting are the basis of the proposed communications architecture.

This basic structure is further specified taking into account requirements of the services in terms of communications, and with 
a special focus on the link between the infrastructure and the final road user: it is the first step in user data collection, as well as the final notification step of service information to the users.

\begin{tabular}{|c|c|}
\hline Parameter & Description \\
\hline Latency & $\begin{array}{l}\text { Aspect related to the time elapsed between the occurrence of an } \\
\text { incident and the reception on part of the user of the correspon- } \\
\text { ding notification. } \\
\text { \{ Real time / Not critical / Irrelevant }\}\end{array}$ \\
\hline Diffusion mode & $\begin{array}{l}\text { Description of the relationship between source and receptor of } \\
\text { information, applicable to any data link } \\
\text { (only a few shall be relevant). } \\
\text { \{ Directivity }\} \text { \{ Unicast / Multicast / GeoCast / BroadCast }\}\end{array}$ \\
\hline Security & $\begin{array}{l}\text { Aspects related to the protection of data and processes against } \\
\text { accidental or deliberate interference. } \\
\text { - Privacy and protection of user data } \\
\text { - Privacy and protection of user communications }\end{array}$ \\
\hline Range & $\begin{array}{l}\text { Aspect related to the normal range of the access technology used } \\
\text { in the link to the road user. } \\
\text { \{Local, short-range / Medium-range / Long-range }\}\end{array}$ \\
\hline Bandwidth & $\begin{array}{l}\text { Aspect related to the volume of the data which will be transferred } \\
\text { through the link, including both typical } \\
\text { and peak values. } \\
\{\text { Low / Medium / Large }\}\end{array}$ \\
\hline
\end{tabular}

Table 1: Communications links requirements parameters. 
In the case of the FOTsis services, the requirements in terms of the parameters listed above resulted in a choice of communication technologies which can be summarised in the following main guidelines:

- At the Access layer, use of a combination of $3 \mathrm{G}+802.11 \mathrm{~b} / \mathrm{g} /$ $\mathrm{n} / \mathrm{p}$

- At the Network layer, use of an IP-based scheme

- At the Application layer, use of web-based services and a generic application deployment platform. Some data exchanges must comply with applicable standards.

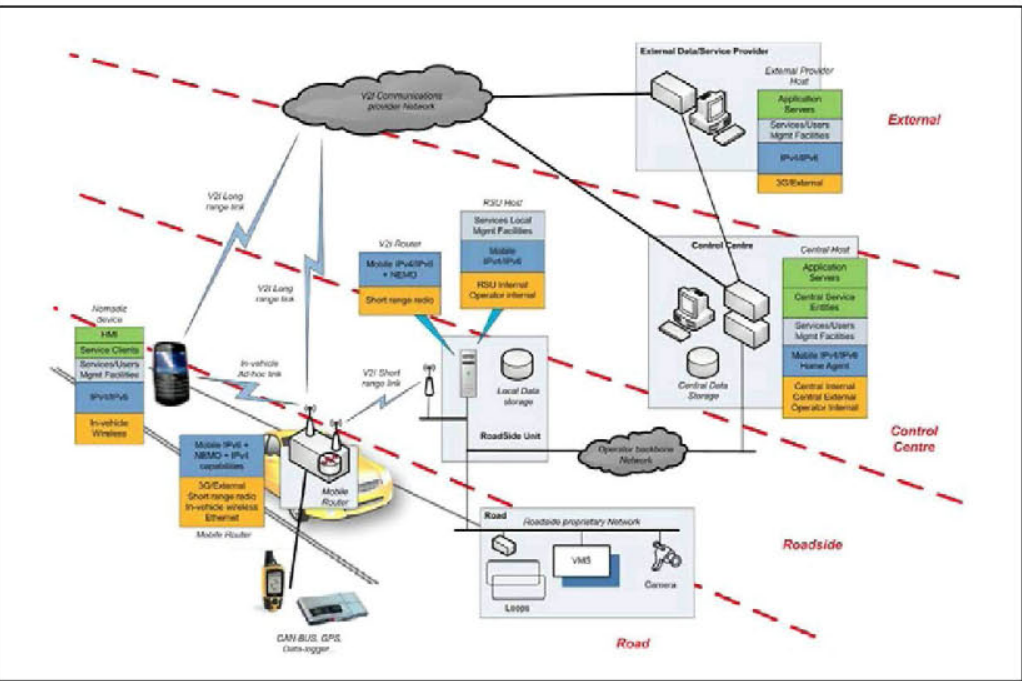

Figure 3: Overall FOTsis architecture deployment. 
The use of a combination of short range and long range radio link with the road users serves to maximise the connectivity time for the services, while exploiting in certain cases specific advantages of either alternative. IEEE 802.11p hotspots can be used as a high-speed localised point-based data collection and dissemination tool (Alfonso, 2012) while $3 \mathrm{G}$ on the other hand allows for a greater coverage.

The need to ensure the connectivity with a diversity of entities and the flexibility of the technology itself were the reasons that led to choice of IP for the FOTsis services deployment. IP includes long established extensions for mobility (IETF Network WG, 2005a, 2010, 2011), and security (IETF Network WG, 2005 b), as well as the capability to operate over other Cooperative ITS network layer initiatives such as GeoNetworking protocols (ETSI TS 102 636-6-1 V1.1.1). FOTsis thoroughly explores the IPv6 developments in the areas mentioned above, using the project's expertise in the field but also through a cooperation with the FP7 ITSSv6 project, which aims to complete, implement and validate IPv6-related ITS Station standards ("ITSSv6 Presentation", n.d.). The use of IPv4 in some of the elements of the FOTsis deployments means that besides IPv6, mechanisms like dualstack and $\mathrm{MCoA}$, amongst others, are also being used.

\subsection{Architecture pilot tests}

Preliminary tests have been conducted in FOTsis for the validation of the architecture, successfully demonstrating data exchanges at IP level from the vehicle on-board devices, both embedded as well as user's mobile devices, to the remote service provider. These tests validate the V2I and I2V communications in $802.11 \mathrm{n} / 802.11 \mathrm{p}$ and $3 \mathrm{G}$ technologies, fully assessing the performance of the technologies in a real deployment scenario. 
Pilot tests have been conducted in Grândola, Baixo Alentejo, Portugal and M12 and A2, Madrid, Spain, using a variety of scenarios, being the most complex:

$\checkmark 2$ on-board Mobile Router and 2802.11 n, 2 802.11p RSUs, for full service connectivity, data collection and service provision with $\mathrm{OBU}$-equipped special vehicle and conventional vehicle via a local mobility manager at the HCC.

Results of the pilot tests so far have been satisfactory, providing the user and the services with a seamless data connection depending on the available technologies and at the 802.11p hotspots, providing around $2 \mathrm{Km}$ coverage with controlled delays and high bandwidth to ensure critical communications.

\section{Impact assessment - Safety issues}

FOTsis is collecting data from the preliminary service and user tests. This data will be afterwards evaluated to see to which extent the designed services achieve the goals proposed. Data is collected regarding travel times and service levels, number of incidents and dangerous weather conditions, driving behaviour and enforcement conditions, amongst many others, to allow a thorough performance analysis of the applications.

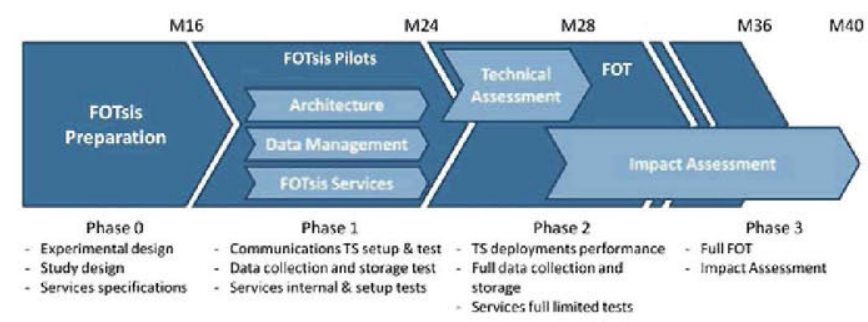

Figure 4: FOTsis project main Phases. 
Service 1 will be tested at the M12 test-site in Spain and Pathe Motorway test-site in Greece. Since Service 1 is oriented to emergency services rather than private vehicles, tests will involve around 5 users, which depending the type of specific test to be conducted, could be road operator users, emergency response teams or both together. Service 2 will be tested at the M12 and AUMECSA testsites in Spain. Service 2 is more oriented to private road users, and therefore a larger number of users will be involved in the tests, with about 50 users being recruited for each test-site. While most of these users will use a $3 \mathrm{G}$-based service provision, some of them will be subject to the detailed tests, in which the full FOTsis communications architecture will be deployed, including $802.11 \mathrm{p}$ equipment, full mobility support, and advanced vehicle tracking and warning notification functionalities. Test plans have been outlined in detailed for the 7 FOTsis services.

The specification of impact assessment procedures in a complex project such as FOTsis is in itself a complex task, including both objective data about the services, the vehicles, the users' trips and all the circumstances surrounding the related events, as well as subjective data complementing this objective information. In the context of FOTsis evaluation procedures and the complexity of the evaluation and the data necessary, the recruitment of users and the collection of user's data is also a key stage in the project. FOTsis works closely with local driver associations to make sure that the recruitment of participants meets the requirements of the project.

For all the 7 FOTsis services, expected benefits have been identified according to their operative details and the proposed deployment architecture, resulting in faster response times, more accurate information for the road operators and users, reduction in congestion levels, and so on. However, for impact evaluation, these expected benefits cannot be addressed directly due to the analysis complexity, and must be divided in simpler topics that can be answered by collection of data from the tests. 
Procedures for analysis depend on the nature of the tested parameter and the objective under evaluation: traffic efficiency, road safety or sustainability, but always following the test conditions defined earlier in the project in terms of Research Questions and Hypotheses (FOT-NET, 2011). These are more specific questions which help to focus on a more direct answer of what are after all very broad objectives, and which in turn allow for a more systematic approach to the tests specification, data collection, impact assessment and the final analysis of the services.

In the case of FOTsis services S1 and S2, assessment of their performance in terms of safety required the formulation of testing procedures addressing specifically road safety parameters. These are collected and aggregated in such a way that the Research Questions related will yield significant results.

\begin{tabular}{|c|c|c|}
\hline$R Q$ Category & RQ Key & Research Question \\
\hline \multirow[t]{2}{*}{ Number of Accidents } & R01 & Is there a change in the number of incidents? \\
\hline & $\mathrm{RO} 2$ & Is there a change in the severity of accidents? \\
\hline Travel time & R05 & Is there a change in travel time? \\
\hline Traffic volume & R06 & Is there a change in traffic volume? \\
\hline Traffic flow & R08 & Is there a change in traffic conditions? \\
\hline Weather conditions & R011 & $\begin{array}{l}\text { Is there a change in driving in adverse weather } \\
\text { conditions? }\end{array}$ \\
\hline Human factor & $\mathrm{R} 13$ & Do service users accept the recommendations? \\
\hline \multirow[t]{2}{*}{ Resources allocation } & R24 & $\begin{array}{l}\text { Is there a change in the number of unassigned } \\
\text { emergency vehicles? }\end{array}$ \\
\hline & R25 & Is there a change in the emergency response time? \\
\hline Infrastructure design & R27 & $\begin{array}{c}\text { Does the identification of dangerous points } \\
\text { improve? }\end{array}$ \\
\hline
\end{tabular}

Table 2: Selected safety impact assessment FOTsis Research Questions. 
These Research Questions, however, are just the basis for the safety impact assessment to be carried out afterwards. Combined assessment of the data collected in relation to these variables will be performed to analyse, amongst others, the detailed impact of the services in terms of reduction in primary and secondary incidents, the overall travel risk reduction or the efficiency of the emergency resources management.

In the context of safety, different methodologies have been proposed for assessing the safety impact of C-ITS services. FOTsis will apply methods developed in eIMPACT and CODIA projects (Kulmala, R.; Rämä, P.; Sihvola, N., 2008, October), based on a number of assumptions concerning the technical and functional features of the systems as well as their costs and deployment scenarios. A similar initiative, albeit more statistically-based, for impact assessment is used in TeleFOT (TeleFOT, 2010), based on statistic methods applied to significant quantities of data to verify Research Questions and Hypotheses.

Last but not least, , another important point to be assessed is the impact that regional differences, in terms of the regulatory framework and data exchange protocol between public and private entities, might have on the deployment of complex ITS services integrating a number of heterogeneous actors. The FOTsis project offers a unique opportunity to identify the risks involved at a technical, functional and administrative level when deploying advanced complex ITS services in different regional environments, and perhaps an opportunity to advance in the identification of what steps are necessary in order to minimise the effects of those risks. 


\section{Conclusions}

The ultimate goal of Cooperative ITS Services is to use advanced communications technologies to improve surface transport in terms of traffic efficiency, safety and sustainability. In order to achieve these objectives, ITS services must integrate and process information coming from different sources to ensure that they have, at any given time, the most accurate image of the transport environment and the users within that environment. The FOTsis project focuses on the infrastructure side of the environment to exploit its potential as a key element of the road, i.e. as a data collector, processor and actuator.

During the FOTsis execution, data is collected to evaluate the performance of the 7 selected services and assess the extent to which the project's objectives are met. This assessment requires the collection of the maximum amount of information possible about all the events around the activities of any given service and the circumstances regarding all the entities involved in such activities.

But data collection is only the first step of the impact assessment. In particular, safety impact assessment of C-ITS services is a very challenging task, and FOTsis will undertake several approaches, some statistic-based, some detailed analysis-based, to make sure that the results of the analysis, whether for the more safety-related Services 1 and 2, as well as for the rest of the services, yield solid conclusions as to their expected impacts.

\section{Acknowledgement}

This work has been funded under the FOTsis project which is partly financed by the European Commision in the context of the 7th Framework Programme (FP7) for Research and Technological Development (Grant Agreement no. 270447). 


\section{References}

Alfonso, J. (2012) Driving standards, Traffic Technology International, October/November 2012, 52-53.

FOT-NET. (2011) FESTA Handbook, Version 4. Retrieved from http:// www.its.leeds.ac.uk/festa/downloads/

ISO 21217:2010: Intelligent Transport Systems - Communications access for land mobiles (CALM) - Architecture.

ETSI TS 102 636-6-1 V1.1.1: Intelligent Transport Systems (ITS) - Vehicular Communications - GeoNetworking - Part 6: Internet Integration - Sub-part 1: Transmission of IPv6 Packets over GeoNetworking Protocols.

IETF Network WG (2010): RFC 5944: IP Mobility Support for IPv4, Revised.

ITSSv6 Presentation (n.d.) Retrieved January 2013 from http://project. inria.fr/itssv6/

IETF Network WG (2011): RFC 6275: Mobility Support in IPv6.

IETF Network WG (2005a): RFC 3963: Network Mobility (NEMO) Basic Support Protocol.

TeleFOT (2010) Deliverable 4.3.1-Safety Data Analysis Plan Version4.

Kulmala, R.; Rämä, P.; Sihvola, N.(2008, October) Safety Impacts of cooperative systems. 21st ICTCT Workshop Proceedings, Riga, Latvia, 2008 .

IETF Network WG (2005b): RFC 4301: Security Architecture for the Internet Protocol. 